\title{
SUBSPACES OF SMALL CODIMENSION OF FINITE-DIMENSIONAL BANACH SPACES
}

\author{
ALAIN PAJOR AND NICOLE TOMCZAK-JAEGERMANN ${ }^{1}$
}

\begin{abstract}
Given a finite-dimensional Banach space $E$ and a Euclidean norm on $E$, we study relations between the norm and the Euclidean norm on subspaces of $E$ of small codimension. Then for an operator taking values in a Hilbert space, we deduce an inequality for entropy numbers of the operator and its dual.
\end{abstract}

In this note we study the following problem: given an $n$-dimensional Banach space $E$ and a Euclidean norm $\|\cdot\|_{2}$ on $E$ and $0<\lambda<1$, find a subspace $F$ of $E$ with $\operatorname{dim} F \geq \lambda n$ such that

$$
\|x\|_{2} \leq M_{*} f(1-\lambda)\|x\| \text { for } x \in F .
$$

Here $M_{*}$ denotes the Levy mean of the dual norm of $E$ (see the notation below).

This problem was considered by V. Milman, who proved in [18] that estimate (*) holds for a certain exponential function $f$. The estimate was improved later in [10] to $f(1-\lambda) \leq K /(1-\lambda)$, where $K$ is a universal constant. The latter result turned out to be important for various applications (cf. $[\mathbf{1}, \mathbf{1 5}, \mathbf{1 1}, \mathbf{1 9}])$.

The main result of this note proves $(*)$ with the function $f(1-\lambda) \leq K / \sqrt{1-\lambda}$. This estimate, besides being optimal (up to a logarithmic factor), can be used to compare entropy numbers of an operator and its dual for operators taking values in a Hilbert space.

Let us recall some notation.

Let $E$ be an $n$-dimensional Banach space; i.e., $E=\left(R^{n},\|\cdot\|\right)$. Let $[\cdot, \cdot]$ be an inner product on $R^{n}$, and let $\|\cdot\| \cdot \|$ be the associated Euclidean norm on $R^{n}$ defined by $\||x|\|=[x, x]^{1 / 2}$, for $x \in R^{n}$.

Let $B_{E}$ be the closed unit ball in $E$. Set

$$
\|x\|_{*}=\sup \left\{|[x, y]| \mid y \in B_{E}\right\} \quad \text { for } x \in R^{n} .
$$

Clearly, $\left(R^{n},\|\cdot\|_{*}\right)$ can be identified with the dual space $E^{*}$. Let $S=\{x \in$ $\left.R^{n}|||| x|| \mid=1\right\}$, and let $\mu$ be the normalized rotation invariant measure on $S$. Define the Levy means $M$ and $M_{*}$ by

$$
M=\left(\int_{S}\|x\|^{2} d \mu\right)^{1 / 2}, \quad M_{*}=\left(\int_{S}\|x\|_{*}^{2} d \mu\right)^{1 / 2} .
$$

We shall employ a similar notation in a context of symmetric convex bodies. For a closed symmetric convex body $V \subset R^{n}$, by $V^{*}$ we denote the dual body defined by $V^{*}=\left\{x \in R^{n}||[x, y] \mid \leq 1\right.$ for all $\left.y \in V\right\}$.

Received by the editors May 29, 1985.

1980 Mathematics Subject Classification (1985 Revision). Primary 46B20; Secondary 47B10.

${ }^{1}$ Research partially supported by NSERC Grant A8854. 
Note that if $\|\cdot\|_{V}$ is the norm associated to $V$ (i.e., $V=\left\{x \in R^{n} \mid\|x\|_{V} \leq 1\right\}$, then the dual norm $\|\cdot\|_{V^{*}}=\left(\|\cdot\|_{V}\right)_{*}$, as defined by (1), is associated to $V^{*}$. We set $E_{V}=\left(R^{n},\|\cdot\|_{V}\right)$ and $E_{V^{*}}=\left(R^{n},\|\cdot\|_{V^{*}}\right)$, and we denote by $M_{V}$ and $M_{V^{*}}$ the corresponding Levy means.

The main result of this note is

THEOREM 1. Let $E=\left(R^{n},\|\cdot\|\right)$ and let $\|\cdot\|$ be a Euclidean norm on $R^{n}$. For every $0<\lambda<1$ there exists a subspace $F \subset R^{n}$ with $\operatorname{dim} F>\lambda n$ such that

$$
\||x|\| \leq K M_{*}(1-\lambda)^{-1 / 2}\|x\| \text { for } x \in F,
$$

where $K$ is a universal constant.

In particular, we have a corollary for finite-dimensional subspaces of a Banach space of cotype 2. It improves earlier results from $[4,10,18]$ (cf. also [11]).

COROLlaRY 1. Let $X$ be a Banach space of cotype 2 and let $E \subset X$ be an $n$ dimensional subspace. For every $0<\lambda<1$ there exists $F \subset E$ with $m=\operatorname{dim} F>$ $\lambda n$ such that

$$
d\left(F, l_{2}^{m}\right) \leq K C_{2}(X)(1-\lambda)^{-1 / 2} \log \left[C_{2}(X)(1-\lambda)^{-1 / 2}+1\right]
$$

where $C_{2}(X)$ is the Gaussian cotype 2 constant of $X$ and $K$ is a universal constant.

SKETCH OF THE PROOF. It can be shown, using results of [15 and 16] (cf. e.g. $[1,4,10,11,14])$, that the Euclidean norm $\|\mid \cdot\|$ induced on $E$ by the ellipsoid of maximal volume contained in $B_{E}$ satisfies $\|x\| \leq\|x\| \|$ for $x \in E$ and $M_{*} \leq K^{\prime} C_{2}(X) \log \left[d\left(E, l_{2}^{n}\right)+1\right]$. Combining this fact either with Milman's iteration procedure (cf., e.g., $[\mathbf{1}, \mathbf{4}, \mathbf{1 0}]$ ) or with an argument from [10] (proof of Theorem 1 and Remark after Theorem 8), yields the result.

The estimate obtained in Theorem 1 and Corollary 1 is, up to a logarithmic factor, the best possible. If $F \subset l_{1}^{n}$ is an $m$-dimensional subspace (with $m>\lambda n$ ), considering a projection $P: l_{1}^{n} \rightarrow l_{1}^{n}$ with $\operatorname{ker} P=F$ and $\gamma_{2}(P) \leq \sqrt{n-m}$, one can easily show that $d\left(F, l_{2}^{m}\right) \geq c(1-\lambda)^{-1 / 2}$, where $c>0$ is an absolute constant.

Theorem 1 can be used to obtain some new estimates between various $s$-numbers of operators from a Banach space $X$ into $l_{2}$. For this let us recall some more notation (cf., e.g., [12]).

For any compact metric space $(T, d)$, we denote by $N(T, d, \varepsilon)$ the smallest number of open balls of radius $\varepsilon$ which cover $T$. If $T$ is the unit ball in an $n$-dimensional Banach space then $N(T,\|\cdot\|, \varepsilon) \leq(1+2 / \varepsilon)^{n}$ (cf., e.g., [6, Lemma 2.4]). Let $X$ and $Y$ be Banach spaces and let $B_{X}$ denote the closed unit ball in $X$. For a compact operator $u: X \rightarrow Y$ between Banach spaces we define the $k$ th entropy number of $n$ by

$$
e_{k}(u)=\inf \left\{\varepsilon>0 \mid N\left(u\left(B_{X}\right),\|\cdot\|_{Y}, \varepsilon\right) \leq 2^{k}\right\} .
$$

Moreover, we define the $k$ th Gelfand number of $u$ by

$$
c_{k}(u)=\inf \left\{\left\|\left.u\right|_{Z}\right\| \mid Z \subset X, \operatorname{codim} Z<k\right\}
$$

and the $k$ th Kolmogorov number by

$$
d_{k}(u)=\inf _{\substack{L \subset Y \\ \operatorname{dim} L<K}} \sup _{x \in B_{X}} \inf _{y \in L}\|u x-y\| .
$$


It is well known (cf., e.g., [12]) that

$$
c_{k}(u)=d_{k}\left(u^{*}\right) .
$$

For the operator $u: l_{2}^{n} \rightarrow X$, we define

$$
l(u)=\left(\int_{R^{n}}\|u x\|^{2} d \gamma_{n}(x)\right)^{1 / 2},
$$

where $\gamma_{n}$ denotes the canonical (normalized) Gaussian measure on $R^{n}$ (cf., e.g., [6]). For any bounded operator $u: l_{2} \rightarrow X$ we set

$$
l(u)=\sup \left\{l(u v) \mid v: l_{2}^{n} \rightarrow l_{2}, v \leq 1, n \in \mathbf{N}\right\} .
$$

If $\operatorname{dim} E=n$ and $u: l_{2}^{n} \rightarrow E$ is one-to-one, then $l(u)=\sqrt{n} M_{V}$, where $V=u^{-1}\left(B_{E}\right)$.

Now we have

Proposition 1. Let $X$ be a Banach space and let $u: X \rightarrow l_{2}$ be a compact operator. Then

$$
\sup _{k} \sqrt{k} c_{k}(u) \leq K l\left(u^{*}\right)
$$

where $K$ is a universal constant.

PROOF. Approximating a given operator by a finite-rank operator and replacing $u$ by $\bar{u}: X / \operatorname{ker} u \rightarrow u(X)$, we may assume that $u: X \rightarrow l_{2}^{n}$ is one-to-one. Define $\|x\|=\left\|u^{-1} x\right\|_{X}$ for $x \in R^{n}$. The conclusion follows from Theorem 1 applied for $E=\left(R^{n},\|\cdot\|\right),\|\| \cdot \|$ the $l_{2}$-norm on $R^{n}$ and $\lambda=1-k / n$.

The next theorem compares entropy numbers of operators $u$ and $u^{*}$ for $u: X \rightarrow l_{2}$. Let us recall that it is still an open problem whether for every compact operator $u: X \rightarrow Y, e_{k}(u)$ and $e_{k}\left(u^{*}\right)$ are asymptotically of the same order, as $k \rightarrow \infty$. For more details on this and related problems and results, cf. $[2,3,8]$.

THEOREM 2. Let $X$ be a Banach space and let $u: X \rightarrow l_{2}$ be a compact operator. Then

$$
\sup _{k} \sqrt{k} e_{k}\left(u^{*}\right) \leq K \sum_{k=1}^{\infty} \frac{e_{k}(u)}{\sqrt{k}}
$$

where $K$ is a universal constant.

The proof of the theorem is based upon two lemmas on entropy numbers. Lemma 1 is an operator version of results on Gaussian processes: the majorization theorem is due to Dudley [5], the minorization theorem to Sudakov [13]. A direct proof of Lemma 1 can be also found in [9].

LEMMA 1. Let $X$ be a Banach space and let $u: X \rightarrow l_{2}$ be a compact operator. Then

(i) $l\left(u^{*}\right) \leq K \sum_{k=1}^{\infty} e_{k}(u) / \sqrt{k}$,

(ii) $\sup _{k} \sqrt{k} e_{k}(u) \leq K l\left(u^{*}\right)$, where $K \geq 1$ is a universal constant.

The next result is due to Carl [2]. 
LEMMA 2. Let $0<p<\infty$. Let $X$ and $Y$ be Banach spaces and let $v: X \rightarrow Y$ be an operator. Then

$$
\sup _{k \leq n} k^{1 / p} e_{k}(v) \leq b_{p} \sup _{k \leq n} k^{1 / p} d_{k}(v) \quad \text { for } n=1,2, \ldots,
$$

where $b_{p}$ depends only on $p$.

Now Theorem 2 follows immediately from Lemma 2, formula (2), Proposition 1 and Lemma 1(i).

Our proof of Theorem 1 is based upon a similar idea to [7]. It will be a consequence of the following technical result.

PROPOSITION 2. There exists $0<d<2^{-7 / 2}$ such that the following is true. Let $|\||\cdot|\|| \mid$ be a Euclidean norm on $R^{n}$ and let $B_{2}$ be the closed unit ball. Let $1 \leq j \leq n$. Let $V$ be a closed symmetric convex body such that

$$
V \subset \bigcup_{z \in \Lambda}(z+d|||z||| W) \cup c B_{2},
$$

with a set $\Lambda \subset R^{n} \backslash\{0\}$ with $\Lambda \leq e^{d^{2} j}$ and some closed symmetric convex body $W$ such that $W \subset B_{2}$ and $M_{W^{*}} \leq \sqrt{j / n}$, and for some constant $c$. Then there exists a subspace $F \subset R^{n}$ with $\operatorname{dim} \bar{F} \geq n-j$ such that

$$
\|x\| \leq c\|x\|_{V} \quad \text { for } x \in F \text {. }
$$

PROOF. Applying an affine transformation, if necessary, we may assume that $\||x|\|=\left(\sum_{i=1}^{n} x_{i}^{2}\right)^{1 / 2}$ for $x \in R^{n}$. Denote by $M_{j}$ the median of the function $\psi_{j}(x)=\left(\sum_{i=1}^{j} x_{i}^{2}\right)^{1 / 2}$ on $S$. It is well known (cf., e.g., [6]) that $M_{j} \geq a \sqrt{j} / n$, where $0<a<\sqrt{2}$ is a universal constant. Set $d=2^{-4} a$. Let $\left\{e_{i}\right\}$ be the standard unit vector basis in $R^{n}$. Let $P_{j}: R^{n} \rightarrow R^{n}$ denote the orthogonal projection onto the subspace spanned by the first $j$ unit vectors.

We need two lemmas. In their formulation $O(n)$ denotes the group of isometries of $l_{2}^{n}$ and $P$ is the Haar measure on $O(n)$. For an operator $T: R^{n} \rightarrow R^{n},\|T: E \rightarrow \dot{F}\|$ denotes the norm of $T$ as an operator from $E$ into $F$.

LEMMA 3. Let

$$
A_{1}=\left\{U \in O(n) \mid\left\|P_{j} U: E_{W} \rightarrow l_{2}^{n}\right\|>8 M_{j} / a\right\}
$$

Then $P\left(A_{1}\right)<\frac{1}{2}$.

LEMMA 4. Let

$$
A_{2}=\left\{U \in O(n)|\exists z \in \Lambda|||\left|P_{j} U z\right|\left\|<\frac{3}{4} M_{j}\right\||| z|| \mid\right\} .
$$

Then $P\left(A_{2}\right)<\frac{1}{2}$.

Assuming the truth of Lemmas 3 and 4 we complete the proof as follows. Pick an isometry $U_{0}: l_{2}^{n} \rightarrow l_{2}^{n}$ such that

$$
\left\|P_{j} U_{0}: E_{W} \rightarrow l_{2}^{n}\right\| \leq 8 M_{j} / a, \quad\left\|\left|P_{j} U_{0} z\right|\right\| \geq\left(3 M_{j} / 4\right)|\|z \mid\| \quad \text { for all } z \in \Lambda .
$$

Then

$$
\left(\operatorname{ker} P_{j} U_{0}\right) \cap(z+d \||| z|| W)=0 \quad \text { for all } z \in \Lambda \text {. }
$$


Indeed, if $y \in\left(\operatorname{ker} P_{j} U_{0}\right) \cap(z+d \||| z|| W)$, then

$$
\begin{aligned}
0 & =\left\|P_{j} U_{0} y\right\|\left|\geq\left\|P_{j} U_{0} z\left|\|-\| P_{j} U_{0}(y-z) \|\right|\right.\right. \\
& \geq\left(3 M_{j} / 4\right)|||z|\left\|-\left(8 M_{j} / a\right)\right\| y-z\left\|_{W} \geq\left(3 M_{j} / 4\right)\left|\left\|z\left|\left\|-\left(8 M_{j} / a\right) d\right\|\right||z|\right\|>0,\right.\right.
\end{aligned}
$$

giving a contradiction. Set $F=\operatorname{ker} P_{j} U_{0}$. From (5) and (3) it follows that $F \cap V \subset$ $c B_{2}$, which is equivalent to (4).

PROOF OF LEMMA 3 . Let $\tilde{\Lambda}$ be a $\frac{1}{2}$-net in $B_{2} \cap\left[e_{1}, \ldots, e_{j}\right]$ with minimal cardinality. Then $|\tilde{\Lambda}|=N\left(B_{2} \cap\left[e_{1}, \ldots, e_{j}\right],\||\cdot|\|, \frac{1}{2}\right) \leq 5^{j}$. Thus

$$
\begin{aligned}
P\left(A_{1}\right) & =P\left\{U \in O(n) \mid\left\|U: l_{2}^{j} \rightarrow E_{W^{*}}\right\|>8 M_{j} / a\right\} \\
& \leq P\left\{U \in O(n)|\exists z \in \tilde{\Lambda}|\|U z\|_{W^{*}}>4 M_{j} / a\right\} \\
& \leq 5^{j} \mu\left\{y \in S \mid\|y\|_{W^{*}}>4 M_{j} / a\right\} .
\end{aligned}
$$

By the isoperimetric inequality (cf. [6, (2.6)], the measure of the latter set is smaller than or equal to

$$
4 \exp \left(-n\left(4 M_{j} / a-M_{W^{*}}\right)^{2} / 2\right) \leq 4 \exp (-9 j / 2) .
$$

Therefore

$$
P\left(A_{1}\right) \leq 4 \cdot 5^{j} \exp (-9 j / 2)<1 / 2 .
$$

Lemma 4 follows immediately from the isoperimetric inequality and the definition of $d$.

Now we are ready to prove Theorem 1 .

ProOF OF THEOREM 1 . Fix $0<\lambda<1$. Let $j$ be the smaller integer larger than or equal to $(1-\lambda) n$. Set $V=\left(d_{1} \sqrt{1-\lambda} / M_{*}\right) B_{E}$, where a constant $0<d_{1}<1$ will be defined later, $W=\left(\sqrt{1-\lambda} / M_{*}\right) B_{E} \cap B_{2}$. Since $W \subset\left(\sqrt{1-\lambda} / M_{*}\right) B_{E}$, the dual bodies satisfy the opposite inclusion and $\left(M_{*} / \sqrt{1-\lambda}\right) B_{E^{*}} \subset W^{*}$. Thus $M_{W^{*}} \leq\left(\sqrt{1-\lambda} / M_{*}\right) M_{*} \leq \sqrt{j / n}$. Notice also that $M_{V^{*}}=\left(d_{1} \sqrt{1-\lambda} / M_{*}\right) M_{*}=$ $d_{1} \sqrt{1-\lambda}$.

Consider the compact metric space $(V,\||\cdot|\| \mid)$. Let $\Lambda$ be a $1 / 2$-net in $V$ with minimal cardinality. It follows from Lemma 1 (ii), applied to the formal identity operator $u:\left(R^{n}, V\right) \rightarrow\left(R^{n},|||\cdot|||\right)$ that

$$
|\Lambda|=N(V,|||\cdot| \mid, \varepsilon) \leq 2^{4 K^{2} n M_{V^{*}}^{2}} \leq \exp \left(d^{2} j\right),
$$

where $K \geq 1$ is the constant from Lemma 1 and $d_{1}=d / 2 K \sqrt{\log 2}$.

To show (3) fix $x \in V$. Pick $z \in \Lambda$ such that $\||x-z|\|<1 / 2$. If $\||x|\| \geq$ $1 / K \sqrt{\log 2}+1 / 2 d+1 / 2$, then $\| z|| \mid \geq 1 / K \sqrt{\log 2}+1 / 2 d$. Therefore,

$$
\begin{aligned}
& x-z \in 2 V=\left(2 d_{1} \sqrt{1-\lambda} / M_{*}\right) B_{E} \subset d\|\| z \mid \|\left(\sqrt{1-\lambda} / M_{*}\right) B_{E}, \\
& x-z \in \frac{1}{2} B_{2} \subset d\|\| z \| B_{2} .
\end{aligned}
$$

Hence $x-z \in d\||| z\| \mid W$. This shows (3) with

$$
c=1 / K \sqrt{\log 2}+1 / 2 d+1 / 2 \text {. }
$$

Now Proposition 2 implies that there exists $F \subset R^{n}$ with $\operatorname{dim} F \geq n-j>\lambda n-1$ such that

$$
\|\| x\|\mid \leq c\| x\left\|_{V}=K^{\prime} M_{*}(1-\lambda)^{-1 / 2}\right\| x \| \quad \text { for } x \in F,
$$

where $K^{\prime}=2 c K \sqrt{\log 2} / d$. 


\section{REFERENCES}

1. J. Bourgain and V. D. Milman, Sections euclidiennes et volume des corps convexes symétriques, C.R. Acad. Sci. Paris Sér. A I Math. 300 (1985), 435-438.

2. B. Carl, Entropy numbers, s-numbers and eigenvalue problem, J. Funct. Anal. 41 (1981), 290-306.

3. __ On Gelfand, Kolmogonov and entropy numbers of operators acting between special Banach spaces (to appear).

4. S. Dilworth and S. Szarek, The cotype constant and almost Euclidean decomposition of finite dimensional normed spaces (to appear).

5. R. J. Dudley, The size of compact subsets of Hilbert space and continuity of Gaussian processes, J. Funct. Anal. 1 (1967), 290-330.

6. T. Figiel, J. Lindenstrauss and V. D. Milman, The dimensions of almost spherical sections of convex bodies, Acta Math. 139 (1977), 53-94.

7. E. D. Gluskin, Norms of random matrices and diameters of finite dimensional sets, Mat. Sb. 120 (1983), 180-189. (Russian)

8. Y. Gordon, H. König and C. Schütt, Geometric and probabilistic estimates for entropy and approximation numbers of operators, J. Approx. Theory (to appear).

9. T. Kühn, $\gamma$-radonifying operators and entropy ideals, Math. Nachr. 107(1982), 53-58.

10. V. D. Milman, Random subspaces of proportional dimension of finite dimensional normed spaces; approach through the isoperimetric inequality, Séminaire d'Analyse Fonctionelle $84 / 85$, Université Paris VI et VII, Paris.

11. V. D. Milman and G. Pisier, Banach spaces with a weak cotype 2 property, Israel J. Math. (to appear).

12. A. Pietsch, Operator ideals, North-Holland, Berlin, 1978.

13. V. N. Sudakov, Gaussian random processes and measures of solid angles in Hilbert space, Soviet Math. Dokl. 12 (1971), 412-415.

14. A. Pajor and N. Tomczak-Jaegermann, Nombres de Gelfand et sections euclidiennes de grande dimension, Séminaire d'Analyse Fonctionelle $84 / 85$, Université Paris VI et VII, Paris.

15. J. Bourgain and V. D. Milman, On Mahler's conjecture on the volume of a convex symmetric body and its polar, Preprint, I.H.E.S.

16. W. J. Davis, V. D. Milman and N. Tomczak-Jaegermann, The distance between certain -dimensional spaces, Israel J. Math. 39 (1981), 1-15.

17. T. Figiel and N. Tomczak-Jaegermann, Projections onto Hilbertian subspaces of Banach spaces, Israel J. Math. 33 (1979), 155-171.

18. V. D. Milman, Volume approach and iteration procedures in local theory of normed spaces, Missouri Conf. 1984.

19. V. D. Milman and G. Pisier, Gaussian processes and mixed volumes, Ann. Probab. (to appear).

20. A. Yu. Garnaev and E. D. Gluskin, On diameters of the Euclidean sphere, Dokl. Akad. Nauk SSSR 277 (1984), 1048-1052.

Université des SCIENCES et TeChNiques DE Lille, U.E.R. DE Mathématiques, 59655 Villeneuve D'ASCQ Cedex, France

Department of Mathematics, University of Alberta, Edmonton, Alberta T6J 2G1, CANADA 